\title{
VALIDACIÓN DEL SCANNING NEUROPSICOLÓGICO DE LA PERSONALIDAD EN ESTUDIANTES UNIVERSITARIOS
}

Validation of scanning of neuro psychological the personality in university students

\author{
César Sarria ${ }^{1}$, Victoria llaja, Pedro García, Marina. Salazar \\ Universidad Nacional Mayor de San Marcos, Lima, Perú \\ (ReCiBIdo el 05/01/2011 - AcEPtado el 08/05/2011)
}

\begin{abstract}
RESUMEN
La escala Scanning Neuropsicológico de la Personalidad, elaborado por C. Sarria, V. Llaja, P. García, M. Salazar, G. Salazar (2009), es un reciente instrumento inédito diseñado para examinar los componentes estructurales del Sistema de la Personalidad en estudiantes universitarios, basado en el Modelo Teórico Informacional (Ortiz, 1994, 1997, 2004a, 2004b). Mediante el estudio reportado en el presente artículo, se obtuvieron evidencias que señalan que este instrumento posee validez de contenido y de constructo y demostró un índice alto de confiabilidad en lo que respecta tanto a su consistencia interna como en el análisis factorial de todos sus ítems. Finalmente, se realizó el análisis cualitativo neuropsicológico para que pueda ser empleado posteriormente en el examen neuropsicológico de muestras clínicas y educacionales.
\end{abstract}

Palabras clave: Validez de contenido, validez de constructo, Scanning Neuropsicológico, Análisis factorial, modelo teórico informacional.

\begin{abstract}
Neuropsychological Scanning scale of Personality, prepared by C. Sarria, V. Llaja, P. García, M. Salazar, G. Salazar (2009) is a recent unpublished instrument designed to examine the structural components of the Personality System in university students, based on the theoretical model Informational (Ortiz, 1994, 1997, 2004a, 2004b). Through the study reported in this paper, we obtained evidence indicating that this instrument has content validity and construct and showed a high rate of reliability in terms of both: its internal consistency and factorial analysis on all items. Finally, qualitative analysis was performed neuropsychological so it can be used later in the neuropsychological examination of clinical samples, and educational.
\end{abstract}

Keywords: Content validity, construct validity, neuropsychological scanning, factorial analysis, informational theoretical model.

1 Docente Principal de la Facultad de Psicología de la UNMSM. E-mail: csarria@unmsm.edu.pe 


\section{VISIÓN GENERAL}

Las investigaciones en el campo Neuropsicológico de la realidad nacional no son muy bastas en la actualidad, así mismo no contamos con instrumentos validados en el medio que evalúen los componentes del Sistema de Personalidad, bajo el Modelo Teórico Informacional. En lo que se refiere a instrumentos del área clínica, podemos considerar la Batería Neuropsicológica POC-2005, motivo de publicaciones anteriores en la Revista de la Academia de Neurociencias del Perú (Enero-Abril del 2009) y que demostraron validez clínica en sus hallazgos en el síndrome Kluver-Bucy y en la enfermedad de Parkinson.

Entre otros estudios con el Modelo Informacional tenemos los realizados por Ortiz,P.(1994, 1997, 2004a, 2004b), donde se plantearon definiciones conceptuales tales como: Información Social que explica la actividad personal, la actividad consciente, y la actividad cerebral de los hombres. La redefinición de personalidad descrita por el autor como el individuo total, y cuando este se organiza núclearmente desde el componente del temperamento se dice que se comporta. Cuando se organiza núclearmente desde el componente del Intelecto se desempeña, y cuando se organiza desde el carácter se conduce: de allí que este estudio realizará el análisis interpretativo de los hallazgos basados en el estudio sociobiológico de la personalidad. Por tratarse de un modelo monista materialista, nos permitirá a su vez explicar dentro de una actitud científica humanista, no sólo la naturaleza de la vida, de la sociedad y de los hombres, sino también saber qué son realmente la conciencia y la personalidad, propósito que sólo se comprende por la visión integradora y filosófica que caracteriza los diversos trabajos y publicaciones de su autor.

La validación de este instrumento constituye un primer esfuerzo de tener una herramienta de trabajo clínico para la evaluación de los procesos de la actividad personal planteados por el modelo informacional TIP y se le puede considerar un estudio piloto que servirá de sustento significativo para la proyección de una línea de investigación aplicativa y tecnológica, con la teoría informacional de la Personalidad.

Considerando el potencial beneficio del método psicométrico, en el presente artículo reportaremos un estudio, dentro del marco de validación y confiabilidad de la Escala Screening Neuropsicológico de la Personalidad. Se trabajó con 500 sujetos, 35\% son varones y $64 \%$ son mujeres.

Según la Teoría Clásica de los Test de Muñiz J. (1996), la muestra ideal para conocer la validez y confiabilidad de un instrumento es de un mínimo de 100 participantes.

La prueba tuvo en una primera etapa una cantidad amplia de ítems. Se inició su construcción con 142 ítems, y luego se redujo a 70 ítems.

Pasos:

Hemos trabajado para conocer en primera instancia la confiabilidad, por el método de consistencia interna y se aplicó el coeficiente alfa de Cronback (Cronback,1951), el cual fue de 0.50 , lo que indica que tiene una Confiabilidad Promedio.

El análisis del trabajo se realizó con el SpSS versión 17. 


\section{ANÁLISIS DE LAS ESCALAS}

Para la confiabilidad por áreas:

Se agrupó los ítems, con el mismo procedimiento de la confiabilidad general.

La validez va a ser extraída por Criterio de Jueces (Validez de Contenido).

Se utilizó la formúla de V de Aiken de Ezcurra, pero adaptada en la versión de Programa computarizado de C. Merino y J. Livia, de Intervalos de confianza asimétricos (Visual Basic para la V de Aiken España- Murcia: Anales de Psicología, 2009).

Luego, se procedió a realizar el Análisis Factorial. Antes de ello se hizo la Validez de Contenido para dar más peso estadístico a la prueba.

Más adelante se ejecutarán en un estudio posterior otros tipos de validez y de confiabilidad.

En cuanto a la baremación se aplicó a una muestra representativa de estudiantes universitarios tanto de Universidades estatales como privadas.

Por el momento estamos efectuando sólo un estudio exploratorio en esta muestra representativa de estudiantes universitarios.

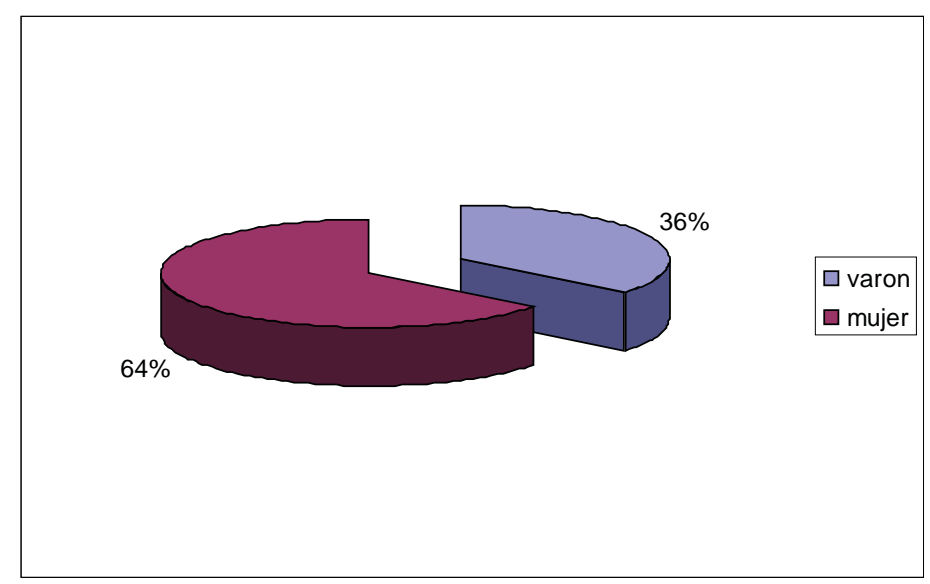

FIGURA 1.

Parental (Lovejoy et al., 1999). Del primer estudio, nuestros objetivos descansan en la replicabilidad de la estructura interna del inventario. Junto con ello, la estimación de la consistencia interna también será evaluada. En el segundo estudio, extenderemos las evidencias de validez con el análisis correlacional de las dimensiones de la crianza con el reporte de las madres sobre aspectos emocionales, relacionales con la pareja actual y evaluación general del vecindario. Estos dos estudios abordan una de las estrategias de evaluación diseñadas por Lovejoy, que es el método de auto-reporte, pretendiendo iniciar posteriores estudios sobre los restantes métodos que ella propuso. Los constructos evaluados por este método son dos amplios aspectos de la crianza: las conductas de hostilidad/coerción y de apoyo/compromiso. 


\section{Estudio 1}

\section{Muestra}

Las participantes de este estudio fueron 148 adultos apoderados de niños de entre 5 y 6 años de edad.

Los ítems del Scanning quedaron distribuidos en 3 áreas, y cada uno de ellos se registra a continuación en la Tabla 1:

TABLA 1. Ítems cualificados por área

\begin{tabular}{cccc}
\hline Área & Temperamento & Intelecto & Carácter \\
\hline \multirow{2}{*}{ Ítem } & $1,2,4,5,7,9,11,12$ & $3,6,8,10,15,19$ & $14,21,25,26,34,35$ \\
& $13,16,17,18,20,22$ & $24,29,32,36,38$ & $37,49,41,43,45,47$ \\
& $23,27,28,30,31,33$ & $42,49,51,53,56$ & $48,52,54,55,58,59$ \\
& $40,44,46,50$, & $57,60,62,64,66$ & $61,63,65,68,69$ \\
\hline Total ítem & 24 & $67,70$. & 23 \\
\hline
\end{tabular}

En un análisis interítems se ve la relación que hay entre los ítems y qué es lo que aporta el ítem al constructor. En una prueba específica se espera que la correlación entre ítems sea de 0.25 hasta 0.40 , en una prueba general se espera que la correlación sea de 0.40 hasta 0.50 . Se planteó el siguiente problema:

¿En que medida el análisis psicométrico y cualitativo del Scanning Neuropsicológico de la personalidad arroja resultados válidos y confiables en una muestra de validación de estudiantes universitarios?

\section{HIPÓTESIS}

En cuanto a los planteamientos de hipótesis tenemos:

\section{Hipótesis General}

El análisis psicométrico del Scanning Neuropsicológico de la personalidad arrojará índices de validez y confiabilidad suficientes para validar el instrumento en una muestra de estudiantes universitarios

\section{Hipótesis Específicas}

1. El análisis psicométrico del Scanning Neuropsicológico de la personalidad evalúa de manera confiable los procesos afectivo-emotivos del Temperamento.

2. El análisis psicométrico del Scanning Neuropsicológico de la personalidad evalúa de manera confiable los procesos cognitivo-productivos del Intelecto.

3. El análisis psicométrico del Scanning Neuropsicológico de la personalidad evalúa de manera confiable los procesos conativo-volitivos del Carácter. 


\section{OBJETIVOS}

1. Estimar la validez y confiabilidad del Scanning Neuropsicológico de la Personalidad.

2. Estimar la validez y confiabilidad de los 3 componentes del Sistema de Personalidad, a través del Scanning Neuropsicológico.

3. Desarrollar a su vez un análisis cualitativo neuropsicológico de los 3 componentes estructurales de la personalidad basado en el Modelo Teórico Informacional.

\section{Metas específicas}

1. Contar con un instrumento validado en nuestra realidad, para la evaluación integral de la personalidad.

2. Evaluar los componentes estructurales de la personalidad: Temperamento, Intelecto y Carácter, a fin de tipificar las características predominantes en la muestra de estudio.

3. Obtener un análisis cualitativo, de corte neuropsicólogico, para la interpretación de resultados, bajo el Modelo Informacional de la Personalidad (Ortiz, 1994).

\section{MÉTODO}

La presente investigación es de tipo tecnológica, según la clasificación de Carrasco Díaz (2006). Se empleó un diseño descriptivo-psicométrico, que ayudó a evaluar las propiedades cuantitativas y psicométricas del Scanning Neuropsicológico de la Personalidad. La muestra fue aleatoria representativa, estuvo conformada por 500 estudiantes universitarios de Universidades nacionales y privadas, de grupos etáreos de 20 a 30 años de edad y el criterio de exclusión fue que no sean de la especialidad de Psicología, y sin antecedentes de enfermedad neurológica y/o mental.

Es un trabajo exploratorio, para determinar si nuestro instrumento mide lo que pretende medir.

La primera fase consistió en los procedimientos de validación: empezamos por la validez de contenido de la tabla de consistencia mediante el criterio de expertos, luego se elaboró el test piloto (142 ítems), el cual fue aplicado para la validación semántica de los ítems.

Se elaboró la prueba definitiva, con 70 ítems, que fueron clasificados según el criterio de los expertos de la siguiente manera: 24 de Temperamento, 23 de Intelecto, y 23 de Carácter, la misma que fue aplicada a la muestra total de estudio.

Hemos trabajado para conocer en primera instancia la confiabilidad, a través del Método de consistencia Interna y hemos aplicado el alfa de Cronbach, el cual fue de 0.50 , lo que indica que tiene confiabilidad alta. Además se calcularon las correlaciones interítems a través de la medida que la correlación interítems promedio determinó la homogeneidad dentro de la escala. Las correlaciones promedio entre los ítems estuvieron entre $0.15 \mathrm{y}$ 0.50 (Clark \& Watson, 1995). 
Para la confiabilidad de las Escalas: según la clasificación trifactorial; se va a agrupar los ítems, con el mismo procedimiento de la confiabilidad general. La validez va a ser extraída por el criterio de Jueces, expertos en Neuropsicología clínica y conocedores de la Teoría Informacional (Validez de contenido) orientada al conocimiento de la variabilidad y la estructura de los componentes. Se analizó la redacción de los ítems, vale decir sus estructura semántica y su contenido. Se tomó en cuenta el nivel académico de cada uno de los expertos, que tomaron en cuenta los siguientes parámetros:

A. Ítems que debían ser mejorados en su redacción.

B. Cantidad de ítems que fueron observados y modificados por cada experto.

C. La mayor coincidencia de los jueces en un determinado ítem.

D. Términos poco frecuentes en el idioma castellano.

E. Observaciones y sugerencias.

Luego de lo cual se realizó la fórmula V de Aiken en la versión de Programa computarizado de C. Merino \& J. Livia de Intervalos de confianza asimétricos (Visual Basic para V de Aiken España-Murcia: Anales de Psicología, 2009).

Luego se procedió a analizar el Análisis Factorial , y posteriormente otros tipos de validez y confiabilidad.

\section{RESULTADOS}

\section{Scale: Temperamento}

\begin{tabular}{|c|c|c|c|c|}
\hline \multicolumn{5}{|c|}{ Reliability Statistics } \\
\hline \multicolumn{2}{|c|}{ Cronbach's Alpha } & & $\begin{array}{l}\text { on } \\
\text { Items }\end{array}$ & $\mathrm{N}$ of Items \\
\hline \multicolumn{2}{|c|}{.715} & & & 24 \\
\hline \multicolumn{5}{|c|}{ Case Processing Summary } \\
\hline & & & $\mathrm{N}$ & $\%$ \\
\hline \multirow{3}{*}{ Cases } & Valic & & 500 & 100.0 \\
\hline & Excl & & 0 & .0 \\
\hline & Total & & 500 & 100.0 \\
\hline
\end{tabular}




\begin{tabular}{|c|c|c|c|c|c|c|c|c|c|c|}
\hline \multicolumn{11}{|c|}{ Summary Item Statistics } \\
\hline & Mean & \multicolumn{2}{|c|}{ Minimum } & Maximum & Range & & $\begin{array}{l}\text { ximum / } \\
\text { nimum }\end{array}$ & Variance & \multicolumn{2}{|c|}{$\mathrm{N}$ of Items } \\
\hline Item Means & 2.125 & \multicolumn{2}{|c|}{1.136} & 3.402 & 2.266 & & 2.995 & .504 & \multicolumn{2}{|c|}{24} \\
\hline Item Variances & .715 & \multicolumn{2}{|c|}{.424} & 1.298 & .874 & & 3.063 & .065 & \multicolumn{2}{|c|}{24} \\
\hline \multicolumn{11}{|c|}{ Intraclass Correlation Coefficient } \\
\hline & \multirow{2}{*}{\multicolumn{2}{|c|}{$\begin{array}{c}\text { Intraclass } \\
\text { Correlation }{ }^{\mathrm{a}}\end{array}$}} & \multicolumn{4}{|c|}{$95 \%$ Confidence Interval } & \multicolumn{4}{|c|}{ F Test with True Value 0} \\
\hline & & & Lo & wer Bound & Upper Bo & & Value & df1 & df2 & Sig \\
\hline Single Measures & & & & .081 & .111 & & 3.511 & 499 & 11477 & .000 \\
\hline Average Measures & & & & .678 & .750 & & 3.511 & 499 & 11477 & .000 \\
\hline \multicolumn{11}{|c|}{$\begin{array}{l}\text { Two-way mixed effects model where people effects are random and measures effects are fixed. } \\
\text { a. Type } \mathrm{C} \text { intraclass correlation coefficients using a consistency definition-the between-measure variance is excluded from } \\
\text { the denominator variance. } \\
\text { b. The estimator is the same, whether the interaction effect is present or not. } \\
\text { c. This estimate is computed assuming the interaction effect is absent, because it is not estimable otherwise. }\end{array}$} \\
\hline
\end{tabular}

\section{Scale: Intelecto}

\begin{tabular}{clcc}
\hline \multicolumn{3}{c}{ Case Processing Summary } \\
\hline \multirow{3}{*}{ Cases } & \multicolumn{1}{c}{$\mathrm{N}$} & $\%$ \\
\cline { 2 - 4 } & Valid & 500 & 100.0 \\
\cline { 2 - 4 } & Excluded $^{\mathrm{a}}$ & 0 & .0 \\
\cline { 2 - 4 } & Total & 500 & 100.0 \\
\hline
\end{tabular}

a. Listwise deletion based on all variables in the procedure.

\begin{tabular}{ccc}
\hline & & \\
\hline & Reliability Statistics & \\
\hline Cronbach's Alpha & $\begin{array}{l}\text { Cronbach's } \\
\text { Alpha Based on } \\
\text { Standardized Items }\end{array}$ & N of Items \\
\hline .787 & \multicolumn{1}{c}{.810} & 23 \\
\hline
\end{tabular}

\begin{tabular}{lccccccc}
\hline \multicolumn{8}{c}{ Summary Item Statistics } \\
\hline & Mean & Minimum & Maximum & Range & $\begin{array}{c}\text { Maximum / } \\
\text { Minimum }\end{array}$ & Variance & $\begin{array}{c}\text { N of } \\
\text { Items }\end{array}$ \\
\hline Item Means & 2.731 & 1.816 & 3.444 & 1.628 & 1.896 & .144 & 23 \\
\hline Item Variances & .808 & .420 & 1.742 & 1.322 & 4.150 & .123 & 23 \\
\hline
\end{tabular}




\begin{tabular}{|c|c|c|c|c|c|c|c|}
\hline \multicolumn{8}{|c|}{ Intraclass Correlation Coefficient } \\
\hline & \multirow{2}{*}{$\begin{array}{c}\text { Intraclass } \\
\text { Correlation }^{\mathrm{a}}\end{array}$} & \multicolumn{2}{|c|}{ 95\% Confidence Interval } & \multicolumn{4}{|c|}{ F Test with True Value 0} \\
\hline & & Lower Bound & Upper Bound & Value & df1 & df2 & Sig \\
\hline Single Measures & $.138^{\mathrm{b}}$ & .120 & .159 & 4.692 & 499 & 10978 & .000 \\
\hline Average Measures & $.787^{\mathrm{c}}$ & .759 & .813 & 4.692 & 499 & 10978 & .000 \\
\hline \multicolumn{8}{|c|}{$\begin{array}{l}\text { Two-way mixed effects model where people effects are random and measures effects are fixed. } \\
\text { a. Type } \mathrm{C} \text { intraclass correlation coefficients using a consistency definition-the between-measure variance is excluded from } \\
\text { the denominator variance. } \\
\text { b. The estimator is the same, whether the interaction effect is present or not. } \\
\text { c. This estimate is computed assuming the interaction effect is absent, because it is not estimable otherwise. }\end{array}$} \\
\hline
\end{tabular}

\section{Scale: Caracter}

\begin{tabular}{llcc}
\hline \multicolumn{4}{c}{ Case Processing Summary } \\
\hline \multirow{3}{*}{ Cases } & \multicolumn{1}{c}{$\mathrm{N}$} & $\%$ \\
\cline { 2 - 4 } & Valid $^{*}$ & 500 & 100.0 \\
\cline { 2 - 4 } & Excluded $^{\mathrm{a}}$ & 0 & .0 \\
\cline { 2 - 4 } & Total & 500 & 100.0 \\
\hline
\end{tabular}

a. Listwise deletion based on all variables in the procedure.

\begin{tabular}{|c|c|c|}
\hline \multicolumn{3}{|c|}{ Reliability Statistics } \\
\hline Cronbach's Alpha & $\begin{array}{l}\text { Cronbach's } \\
\text { Alpha Based on } \\
\text { Standardized Items }\end{array}$ & $\mathrm{N}$ of Items \\
\hline .760 & .806 & 23 \\
\hline
\end{tabular}

\begin{tabular}{lccccccc}
\hline \multicolumn{10}{c}{ Summary Item Statistics } \\
\hline & Mean & Minimum & Maximum Range & $\begin{array}{c}\text { Maximum / } \\
\text { Minimum }\end{array}$ & Variance & $\begin{array}{c}\text { N of } \\
\text { Items }\end{array}$ \\
\hline Item Means & 2.740 & 1.292 & 3.344 & 2.052 & 2.588 & .396 & 23 \\
\hline Item Variances & .816 & .387 & 1.679 & 1.292 & 4.337 & .154 & 23 \\
\hline
\end{tabular}

\begin{tabular}{|c|c|c|c|c|c|c|c|}
\hline \multicolumn{8}{|c|}{ Intraclass Correlation Coefficient } \\
\hline & \multirow{2}{*}{$\begin{array}{c}\text { Intraclass } \\
\text { Correlation }^{\mathrm{a}}\end{array}$} & \multicolumn{2}{|c|}{$95 \%$ Confidence Interval } & \multicolumn{4}{|c|}{ F Test with True Value 0} \\
\hline & & Lower Bound & Upper Bound & Value & df1 & df 2 & Sig \\
\hline Single Measures & $.121^{\mathrm{b}}$ & .105 & .140 & 4.171 & 499 & 10978 & .000 \\
\hline Average Measures & $.760^{c}$ & .729 & .790 & 4.171 & 499 & 10978 & .000 \\
\hline \multicolumn{8}{|c|}{$\begin{array}{l}\text { Two-way mixed effects model where people effects are random and measures effects are fixed. } \\
\text { a. Type } \mathrm{C} \text { intraclass correlation coefficients using a consistency definition-the between-measure variance is excluded from } \\
\text { the denominator variance. } \\
\text { b. The estimator is the same, whether the interaction effect is present or not. } \\
\text { c. This estimate is computed assuming the interaction effect is absent, because it is not estimable otherwise. }\end{array}$} \\
\hline
\end{tabular}




\section{Scale: Total Test}

\begin{tabular}{|c|c|c|c|}
\hline \multicolumn{4}{|c|}{ Case Processing Summary } \\
\hline & & $\mathrm{N}$ & $\%$ \\
\hline \multirow{3}{*}{ Cases } & Valid & 500 & 100.0 \\
\hline & Excluded $^{\mathrm{a}}$ & 0 & .0 \\
\hline & Total & 500 & 100.0 \\
\hline
\end{tabular}

\begin{tabular}{ccc}
\hline \multicolumn{3}{c}{ Reliability Statistics } \\
\hline \multirow{2}{*}{ Cronbach's Alpha } & $\begin{array}{l}\text { Cronbach's } \\
\text { Alpha Based on } \\
\text { Standardized Items }\end{array}$ & N of Items \\
\hline .855 & .869 & 70 \\
\hline
\end{tabular}

\begin{tabular}{lccccccc}
\hline \multicolumn{8}{c}{ Summary Item Statistics } \\
\hline & Mean & Minimum & Maximum & Range & $\begin{array}{c}\text { Maximum / } \\
\text { Minimum }\end{array}$ & Variance & $\begin{array}{c}\text { N of } \\
\text { Items }\end{array}$ \\
\hline Item Means & 2.526 & 1.136 & 3.444 & 2.308 & 3.032 & .425 & 70 \\
\hline Item Variances & .779 & .387 & 1.742 & 1.355 & 4.499 & .112 & 70 \\
\hline
\end{tabular}

\begin{tabular}{lcccccccc}
\hline \multicolumn{7}{c}{ Intraclass Correlation Coefficient } \\
\hline & \multirow{2}{*}{$\begin{array}{c}\text { Intraclass } \\
\text { Correlation }\end{array}$} & \multicolumn{2}{c}{$95 \%$ Confidence Interval } & \multicolumn{2}{c}{ F Test with True Value 0 } \\
\cline { 3 - 9 } & & Lower Bound & Upper Bound & Value & df1 & df2 & Sig \\
\hline Single Measures & $.078^{\mathrm{b}}$ & .068 & .089 & 6.889 & 499 & 34431 & .000 \\
\hline Average Measures & $.855^{\mathrm{c}}$ & .836 & .872 & 6.889 & 499 & 34431 & .000 \\
\hline
\end{tabular}

Two-way mixed effects model where people effects are random and measures effects are fixed.

a. Type $\mathrm{C}$ intraclass correlation coefficients using a consistency definition-the between-measure variance is excluded from the denominator variance.

b. The estimator is the same, whether the interaction effect is present or not.

c. This estimate is computed assuming the interaction effect is absent, because it is not estimable otherwise.

En la sección Anexos (1-2-3) podemos observar las correlaciones de los componentes de la personalidad en detalle.

\section{Instrumento}

Escala Scanning Neuropsicológicode la Personalidad (SNP). El diseño de su construcción siguió un cuidadoso procedimiento reportado en Lovejoy et al. (1999), que apuntaba 
hacia la evaluación del Sistema de Personalidad en sus tres componentes: Temperamento, Intelecto y Carácter. El Scanning contiene 70 ítems, seleccionados bajo el Modelo teórico informacional de la Personalidad, donde los examinados deben responder sobre una escala de 0 (Nunca) hasta el 4 (Muy a menudo ) y en alguno de los tres marcos de tiempo: conducta de a menudo, de vez en cuando, y casi nunca. En la validación de contenido, expertos en el modelo Teórico informacional de la Personalidad, utilizado en el marco de la Neurociencias aplicado a la clínica y Educación, comunicaron la relevancia y representatividad de los ítems, que en una escala del 1 al 5, tuvieron índices promedio de 4.28 y 4.51 para cualificar los componentes estructurales de la Personalidad: Temperamento, Intelecto y Carácter.

La validez factorial se evaluó con sucesivas aplicaciones del análisis factorial confirmatorio.

El alfa de Cronbach reportado para temperamento fue de .715, con una correlación inter-ítem promedio de .32 y un rango desde .09 a .52. Para el Intelecto fue de .787, y para el Carácter .760. Para la prueba global el alfa de Cronbach fue de .855.

\section{Procedimiento}

Los investigadores organizaron y ejecutaron un proceso de evaluación a la muestra de estudiantes universitarios de la especialidad de Tecnología Médica, de Educación y Letras del segundo y tercer año de dos universidades: una pública y una privada. Este proceso de evaluación se hizo en el mismo periodo, durante los meses de abril a setiembre del 2010. Los estudiantes fueron evaluadas en grupos de 10 a 15 personas en un salón exento de elementos distractores, paralelamente eran evaluados en otro salón otro grupo con la misma cantidad de examinados. La escala se administró previa explicación de las instrucciones del protocolo de respuesta y así mismo de las probabilidades de respuesta adjuntas a la Escala. Se recomendaba que no olvidaran de colocar los datos demográficos solicitados. Uno de los autores supervisó las respuestas de los estudiantes.

La validez factorial utilizó el análisis factorial exploratorio y el análisis factorial confirmatorio (AFC), consecutivamente. El primero sirvió para revelar los potenciales factores que podrían emerger sin imponer alguna estructura. Luego, el AFC permitirá una prueba directa de la estructura previamente definida por Lovejoy et al. (1999); se aplicó para ello el programa Statistica (StatSoft, 1995). Para evaluar el ajuste del modelo, utilizamos varios criterios de ajuste como lo sugiere Hair, Anderson, Tatham \& Black (1999).

El examen del ajuste del modelo se inicia con el estadístico II2 , pero este comúnmente tiende a rechazar los modelos propuestos asumiendo una sobre-identificación de las especificaciones del modelo (Mulaik, 1993), así que la práctica prefiere el uso complementario de criterios alternativos. Teniendo como referencia los mismos criterios elegidos por Lovejoy y colaboradores, se eligió el error de aproximación raíz media cuadrática (RMSEA), el índice de bondad de ajuste (GFI) y el índice de ajuste comparativo (CFI); adicionalmente se calculará el Índice comparativo de no centralidad (RNI), que aunque no es reportado por Lovejoy, se puede calcular con sus datos publicados.

Todos ellos que indican el grado de ajuste absoluto del modelo, aunque no hay criterios unívocos, los niveles de aceptación de estos índices se hallaron de acuerdo a lo sugerido por Hair et al. (1999). La confiabilidad por consistencia interna será evaluada por el 
coeficiente alfa de Cronbach (Cronbach, 1951); finalmente, la homogeneidad interna será reportada por las correlaciones ítem-test corregida por espuriedad (Thorndike, 1989) y la correlaciones interítems, este último reportado por Lojevoy, et al. (1999).

\section{CONCLUSIONES}

El alfa de Cronbach reportado para temperamento fue de .715 , con una correlación interítems promedio de .32 y un rango desde .09 a .52. Para el Intelecto fue de .787, y para el Carácter .760. Para la prueba global el alfa de Cronbach fue de .855 .

Finalmente, el análisis psicométrico del Scanning Neuropsicológico de la Personalidad arrojó índices de validez y confiabilidad suficientes para validar el instrumento en una muestra de estudiantes universitarios

\section{REFERENCIAS BIBLIOGRÁFICAS}

1. Allport, G. (1973). La personalidad, su configuración y desarrollo. Barcelona: Editorial Herder. España.

2. Allport, G. (1966). Psicología de la personalidad. Buenos Aires: Paidos.

3. Bannister, D. (1969). Una nueva teoría de la personalidad. Barcelona: Fontanella.

4. Barker, C., Pistrang, N., \& Elliot, R. (1994). Research methods in clinical and couseling psychology. West Sussex: John Wiley \& Sons.

5. Carrasco Díaz, S. (2006). Metodología de la Investigación científica. Lima: Editorial San Marcos.

6. Catell, R. (1972). El estudio científico de la personalidad. Mexico: Trillas.

7. Cronbach, L. J. (1951). Coefficient alpha and the internal structure of test. Psychometrika, 16(3), 297-334.

8. Cronbach, L. J., \& Meehl, P. E. (1955). Construct validity in psychological test. Psychological Bulletin, 52, 281-302.

9. Cueli, J.; Reidi, L. (1972). Teorías de la Personalidad. Mexico: Trillas.

10. Díaz Guerrero, R. (1976). Hacia una personalidad social del Tercer Mundo. México: UNAM Cuaderno de Humanidades.

11. Gómez, M.A. (2000). Análisis de contenido cualitativo y cuantitativo:definición, clasificación y metodología. Colombia: Ciencias Humanas, Revista(20) Revisado en 2005. Disponible en: http://www.utp.edu.co/chumanas/revistas/revistas/rev20/ gomez.htm

12. Eysenck, H.J. (1973). El estudio científico de la personalidad. Barcelona: Fontanella.

13. Eysenck, H.J. (1978). Fundamentos biológicos de la personalidad. Barcelona: Fontanella. 
14. Hair, J.F., Jr. Anderson,R.E. Tatham, R. L., \& Black, W. C. (1999). Análisis multivariante. 5. ${ }^{\mathrm{a}}$ ed. Madrid: Prentice Hall Iberia.

15. Hernández R. et al. (2010). Metodología de la investigación. México: McGraw Hill Interamericana Editores.

16. Huberty, C. J. (2003). Multiple correlation versus multiple regression. Educational and Psychological Measurement, 63(2), 271-278.

17. Huberty,C. J. \& Petoskey, M. D. (1999). Use of multiple correlation analysis and multiple regression analysis. Journal of Vocational Education Research, 24; 15-43.

18. Jiménez L. (2002). Construcción y validación de la Escala Psiconeurológica en niños aplicada a la familia. Revista Cultura 16 Año XX. Asociación de Docentes de la UPSMP, pp. 343-369.

19. Llaja,V. (2006). Rasgos de Personalidad y el Síndrome de Burnout en el Profesional de Cuidados Intensivos en un Hospital de EsSalud de Lima. Tesis Doctoral en Psicología. Lima: UPSMP.

20. Muñiz, J. (2006). Teoría clásica de los Test. Madrid: Pirámide.

21. Nunnally, J. C. \& Bernstein, I. J. (1995). Teoría psicométrica. 3. ${ }^{a}$ ed. México, D. F: McGraw Hill

22. Ortiz.P. (2004). El nivel consciente de la actividad personal. Cuadernos de Psicobiología Social 6. Lima: Talleres Gráficos del Centro Editorial UNMSM.

23. Ortiz.P.(2004). Introducción a una Psicobiología del Hombre. Cuadernos de Psicobiología Social 1. Lima: Talleres Gráficos del Centro Editorial UNMSM.

24. Ortiz, P. (1996). La formación de la Personalidad. Lima: Dimaso Editores.

25. Ortiz, P. (1994). El sistema de la Personalidad. Lima: Centro Gráfico comercial Orión.

26. Sarria, C., Llaja, V.\& García, P. (2010). Batería Neuropsicológica: Estudio de la actividad psíquica personal [en prensa]. Lima: Editorial San Marcos.

27. Sarria, C. Llaja, V.\& García, P. (2008). Compendio de Neuropsicología clínica. Lima: Editorial San Marcos.

28. StatSoft, Inc. (1995). STATISTICA for Windows [Computer program manual]. Tulsa, OK: StatSoft, Inc.

29. Thorndike, R. L. (1989). Psicometría Aplicada. México, D. F: Limusa. 